Journal of Universal Mathematics

Vol.3 No.1 PP.33-45 (2020)

ISSN-2618-5660

\title{
INTUITIONISTIC FUZZY HEAT-LIKE EQUATIONS
}

\author{
A.HARIR, S. MELLIANI, AND L.S.CHADLI
}

\begin{abstract}
RÉsumÉ. In this paper, the variational iteration method (VIM) is used for finding exact intuitionistic fuzzy solution of the intuitionistic fuzzy heat-like equations with variable coefficients in one and two dimensions. Several examples are given to show the new theorem of the solution. The results obtained in all cases show the reliability and the efficiency of this methods.
\end{abstract}

\section{INTRODUCTION}

The theory of fuzzy sets proposed by Zadeh [12] has showed successful applications in various fields. In fuzzy set theory, the membership of an element to a fuzzy set is a single value between zero and one. But in reality, it may not always be certain that the degree of nonmembership of an element in a fuzzy set is just equal to 1 minus the degree of membership. That is to say, there may be some hesitation degree. So, as a generalization of fuzzy sets, the concept of intuitionistic fuzzy sets was introduced by Atanassov [1].

In this work, our idea is solving heat-like equations with intuitionistic fuzzy parameters via the same strategy as $\alpha$-cuts using Variational Iteration Method VIM.

The VIM proposed by He in [7, is a method of solving linear or nonlinear problems [1] and gives rapidly convergent successive approximations of the exact solution if that last exists.

In comparison with the paper [5, 6, we investigate problems with intuitionistic fuzzy initial value and intuitionistic fuzzy forcing functions, we propose a new theorem for finding the exact intuitionistic fuzzy solutions, witch extended to the solution for the proposed models.

\section{BASIC CONCEPT OF INTUITIONISTIC FUZZY SETS}

Let a set $X$ be fixed. An intuitionistic fuzzy set $\tilde{A}^{i}$ in $X$ is an object having the form $\tilde{A}^{i}=\left\{\left\langle x, \mu_{\tilde{A}^{i}}(x), \nu_{\tilde{A}^{i}}(x)\right\rangle\right\}$, where $\mu_{\tilde{A}^{i}}(x): X \rightarrow[0,1]$ and $\nu_{\tilde{A}^{i}}(x): X \rightarrow[0,1]$ define the degree of memberschip and degree of nonmembership respectively, of the element $x \in X$ to the set $\tilde{A}^{i}$, which is subset of $X$, for every element of $x \in X$, $0 \leq \mu_{\tilde{A}^{i}}(x)+\nu_{\tilde{A}^{i}}(x) \leq 1$. Let $X=\mathbb{R}$

Definition 2.1. Let $\mathbb{I F}=\left\{\tilde{A}^{i} \mid \tilde{A}^{i}: \mathbb{R} \rightarrow[0,1]^{2}\right.$, satisfies (1) - (5) $\}$ :

An intuitionistic fuzzy number $\tilde{A}^{i}$ is

(1) normal i.e there is any $x_{0}, x_{1} \in \mathbb{R}$ such that $\mu_{\tilde{A}^{i}}\left(x_{0}\right)=1$ and $\nu_{\tilde{A}^{i}}\left(x_{1}\right)=1$

(2) convex for the membership function $\mu_{\tilde{A}^{i}}(x)$ i.e

$$
\mu_{\tilde{A}^{i}}\left(\lambda x_{1}+(1-\lambda) x_{2}\right) \geq \min \left(\mu_{\tilde{A}^{i}}\left(x_{1}\right), \mu_{\tilde{A}^{i}}\left(x_{2}\right)\right) \forall x_{1}, x_{2} \in \mathbb{R}, \lambda \in[0,1]
$$

2000 Mathematics Subject Classification. intuitionistic fuzzy solution.

Key words and phrases. Variational Iteration Method, intuitionistic fuzzy solution, the intuitionistic fuzzy heat-like equations. 
(3) concave for non-membership function $\nu_{\tilde{A}^{i}}(x)$ i.e

$$
\nu_{\tilde{A}^{i}}\left(\lambda x_{1}+(1-\lambda) x_{2}\right) \leq \max \left(\nu_{\tilde{A}^{i}}\left(x_{1}\right), \nu_{\tilde{A}^{i}}\left(x_{2}\right)\right) \forall x_{1}, x_{2} \in \mathbb{R}, \lambda \in[0,1]
$$

(4) $\mu_{\tilde{A}^{i}}(x)$ is upper semi-continuous and $\nu_{\tilde{A}^{i}}(x)$ is lower semi-continuous and

(5) $\operatorname{supp}\left(\mu_{\tilde{A}^{i}}, \nu_{\tilde{A}^{i}}\right)=\operatorname{cl}\left\{x \in \mathbb{R}: \nu_{\tilde{A}^{i}}(x)<1\right\}$ is bounded.

Then $\mathbb{I F}$ is called intuitionistic fuzzy space.

Definition 2.2. If $\tilde{A}^{i}$ is an intuitionistic fuzzy number $\alpha$-cut is given by

$$
\tilde{A}^{i}[\alpha]=\left\{A^{+}[\alpha], A^{-}[\alpha] ; \alpha \in[0,1]\right\}
$$

where $A^{-}[\alpha]=\left\{x \in \mathbb{R}: \nu_{\tilde{A}^{i}}(x) \leq 1-\alpha\right\}, \quad A^{+}[\alpha]=\left\{x \in \mathbb{R}: \mu_{\tilde{A}^{i}}(x) \geq \alpha\right\}$.

It is expressed as $\tilde{A}^{i}[\alpha]=\left\{\left[A_{1}^{+}(\alpha), A_{2}^{+}(\alpha)\right],\left[A_{1}^{-}(\alpha), A_{2}^{-}(\alpha)\right] ; \alpha \in[0,1]\right\}$

(i) $A_{1}^{+}(\alpha)$ and $A_{2}^{-}(\alpha)$ will be continuous, monotonic increasing function of $\alpha$

(ii) $A_{2}^{+}(\alpha)$ and $A_{1}^{-}(\alpha)$ will be continuous, monotonic decreasing function of $\alpha$

(iii) $A_{1}^{+}(1)=A_{2}^{+}(1) ; A_{1}^{-}(0)=A_{2}^{-}(0)$.

Definition 2.3. A Triangular Intuitionistic Fuzzy Number (TIFN) $\tilde{A}^{i}$ is an intuitionistic fuzzy in $\mathbb{R}$ with following membership function $\left(\mu_{\tilde{A}^{i}}(x)\right)$ and non-membership function $\left(\nu_{\tilde{A}^{i}}(x)\right)$,

$$
\mu_{\tilde{A}^{i}}(x)=\left\{\begin{array}{ll}
\frac{x-a_{1}}{b_{1}-a_{1}}, & a_{1} \leq x \leq b_{1} \\
\frac{c_{1}-x}{c_{1}-b_{1}}, & b_{1} \leq x \leq c_{1} \\
0, & \text { otherwise. }
\end{array} \text { and } \nu_{\tilde{A}^{i}}(x)= \begin{cases}\frac{b_{1}-x}{b_{1}-a_{1}^{\prime}}, & a_{1}^{\prime} \leq x \leq b_{1} \\
\frac{x-b_{1}}{c_{1}^{\prime}-b_{1}}, & b_{1} \leq x \leq c_{1}^{\prime} \\
1, & \text { otherwise. }\end{cases}\right.
$$

Where $a_{1}^{\prime}<a_{1}<b_{1}<c_{1}<c_{1}^{\prime}$ and $\mu_{\tilde{A}^{i}}(x), \nu_{\tilde{A}^{i}}(x) \leq 0.5$ for $\mu_{\tilde{A}^{i}}(x)=\nu_{\tilde{A}^{i}}(x) \forall x \in \mathbb{R}$. This TIFN is denoted by $\tilde{A}^{i}=\left(a_{1}^{\prime}, a_{1}, b_{1}, c_{1}, c_{1}^{\prime}\right)$

We will write : (1) $\tilde{A}^{i}>0$ if $a_{1}^{\prime}>0,(2) \tilde{A}^{i} \geq 0$ if $a_{1}^{\prime} \geq 0$, (3) $\tilde{A}^{i}<0$ if $c_{1}^{\prime}<0$ and (4) $\tilde{A}^{i} \leq 0$ if $c_{1}^{\prime} \leq 0$. and

$$
A^{+}[\alpha]=\left[a_{1}+\alpha\left(a_{2}-a_{1}\right), a_{3}-\alpha\left(a_{3}-a_{2}\right)\right] \text { and } A^{-}[\alpha]=\left[a_{1}^{\prime}+\alpha\left(a_{2}-a_{1}^{\prime}\right), a_{3}^{\prime}-\alpha\left(a_{3}^{\prime}-a_{2}\right)\right]
$$

For $\tilde{A}^{i}, \tilde{B}^{i} \in \mathbb{I F}$ and $\lambda \in \mathbb{R}$, the addition and scaler-multiplication are defined as follows

$$
\begin{gathered}
\left(\tilde{A}^{i}+\tilde{B}^{i}\right)[\alpha]=\left(A^{+}[\alpha]+B^{+}[\alpha], A^{-}[\alpha]+B^{-}[\alpha]\right) \\
\lambda \tilde{A}^{i}[\alpha]= \begin{cases}\left(\left[\lambda A_{1}^{+}(\alpha), \lambda A_{2}^{+}(\alpha)\right],\left[\lambda A_{1}^{-}(\alpha), \lambda A_{2}^{-}(\alpha)\right]\right) & \lambda \geq 0 \\
\left(\left[\lambda A_{2}^{+}(\alpha), \lambda A_{1}^{+}(\alpha)\right],\left[\lambda A_{2}^{-}(\alpha), \lambda A_{1}^{-}(\alpha)\right]\right), & \lambda<0\end{cases}
\end{gathered}
$$

Theorem 2.4. Let $F: I \rightarrow \mathbb{I F}$ be differentiable. Denote $F^{+}[\alpha]=\left[f_{1}^{+}(t, \alpha), f_{2}^{+}(t, \alpha)\right], F^{-}[\alpha]=\left[f_{1}^{-}(t, \alpha), f_{2}^{-}(t, \alpha)\right]$. Then $f_{1}^{+}(t, \alpha), f_{2}^{+}(t, \alpha), f_{1}^{-}(t, \alpha)$ and $f_{2}^{-}(t, \alpha)$ are differentiable and

$$
\begin{aligned}
& {F^{+\prime}}^{+}[\alpha]=\left[f_{1}^{+\prime}(t, \alpha), f_{2}^{+\prime}(t, \alpha)\right] \\
& F^{-\prime}[\alpha]=\left[f_{1}^{-\prime}(t, \alpha), f_{2}^{-\prime}(t, \alpha)\right]
\end{aligned}
$$

We adopt the general definition of a intuitionistic fuzzy number given in [2, 3, 4, 10, 9].

\section{INTUITIONISTIC FUZZY HEAT-LIKE EQUATIONS}

We consider the heat-like equations in one and two dimensional cases which can be written in the forms 
- One-dimensional [5] :

$$
U_{t}(t, x)+P(x) U_{x x}(t, x)=F(t, x, k)
$$

- Two-dimensional [5] :

$$
\begin{gathered}
U_{t}(t, x, y)+P(x) U_{x x}(t, x, y)+Q(y) U_{y y}(t, x, y)=F(t, x, y, k) \\
\text { or } U_{t}(t, x, y)+Q(y) U_{x x}(t, x, y)+P(x) U_{y y}(t, x, y)=F(t, x, y, k)
\end{gathered}
$$

subject to certain initial and boundary conditions.

These initial and boundary conditions, in state two-dimensional, can come in a variety of forms such as $U(0, x, y)=c_{1}$ or $U(0, x, y)=g_{1}\left(x, y, c_{2}\right)$ or $U\left(M_{1}, x, y\right)=g_{2}\left(x, y, c_{3}, c_{4}\right), \ldots$

In this paper the method is applied for the heat-like equation $(3.2)$. For $(3.1)$ and $(3.3)$, the same discussion can be made. In following lines, the components of $(3.2)$ are enumerated :

- $I_{1}=\left[0, M_{1}\right], I_{2}=\left[M_{2}, M_{3}\right]$ and $I_{3}=\left[M_{4}, M_{5}\right]$ are three intervals, which $M_{n_{1}}\left(n_{1}=2,3,4,5\right)$ is negative or positive and $M_{1}>0$.

- F(t, $x, y, k), U(t, x, y), P(x)$ and $Q(y)$ will be continuous functions for $(t, x, y) \in \prod_{j=1}^{3} I_{j}$.

- $P(x)$ and $Q(y)$ have a finite number of roots for each $(x, y) \in I_{2} \times I_{3}$

$-k=\left(k_{1}, \ldots, k_{n}\right)$ and $c=\left(c_{1}, \ldots, c_{m}\right)$ are vectors of constants with $k_{j} \in J_{j}$ and $c_{r} \in L_{r}$.

Assume that 3.2 has a solution

$$
U(t, x, y)=G(t, x, y, k, c)
$$

for $G$ and

$$
\left((G(t, x, y, k, c))_{t}+P(x)(G(t, x, y, k, c))_{x x}+Q(y)(G(t, x, y, k, c))_{y y}\right.
$$

are continuous with $(t, x, y) \in \prod_{j=1}^{3} I_{j}, k \in J=\prod_{j=1}^{n} J_{j}, \quad$ and $\quad c \in L=\prod_{r=1}^{m} L_{r}$.

The constants $k_{j}$ and $c_{r}$ are not known exactly so there will be uncertainty in their values. We will model this uncertainty using intuitionistic fuzzy numbers. So, we will substitute intuitionistic fuzzy numbers $\tilde{K}_{j}^{i}$ for $k_{j}, \tilde{K}_{j}^{i}$ in $J_{j}, 1 \leq j \leq n$, and substitute intuitionistic fuzzy numbers $\tilde{C}_{r}^{i}$ for $c_{r}, \tilde{C}_{r}^{i}$ in $L_{r}, 1 \leq r \leq m$.

The intuitionistic fuzzy heat-like equation is

$$
\tilde{U}_{t}^{i}(t, x, y)+P(x) \tilde{U}_{x x}^{i}(t, x, y)+Q(y) \tilde{U}_{y y}^{i}(t, x, y)=\tilde{F}^{i}\left(t, x, y, \tilde{K}^{i}\right)
$$

where $\tilde{K}^{i}=\tilde{K}_{1}^{i}, \cdots \tilde{K}_{n}^{i}$ for $\tilde{K}_{j}^{i}$ an intuitionistic fuzzy number in $J_{j}, 1 \leq j \leq n$. The function $U$ where $\tilde{U}^{i}$ maps $\prod_{j=1}^{3} I_{j}$ into intuitionistic fuzzy numbers.

That is, $\tilde{U}^{i}(t, x, y)=\tilde{Z}^{i}$ where $\tilde{Z}^{i}$ is an intuitionistic fuzzy number.

subject to certain initial and boundary conditions. The initial and boundary conditions can be of the form

$$
\tilde{U}^{i}(0, x, y)=\tilde{C}_{1}^{i} \text { or } \tilde{U}^{i}(0, x, y)=\tilde{g}_{1}^{i}\left(x, y, \tilde{C}_{2}^{i}\right) \text { or } \tilde{U}^{i}\left(M_{1}, x, y\right)=\tilde{g}_{2}^{i}\left(x, y, \tilde{C}_{3}^{i}, \tilde{C}_{4}^{i}\right)
$$

The $\tilde{g}_{j}^{i}$ is the model intuitionistic fuzzy function of $g_{j}$. Let $\tilde{C}^{i}=\tilde{C}_{1}^{i}, \cdots \tilde{C}_{n}^{i}$ with $\tilde{C}^{i}$ triangular intuitionistic fuzzy number in $L_{r}, 1 \leq r \leq m$. We wish to solve the problem given in 3.5 . Let $\tilde{Z}^{i}(t, x, y)=\tilde{G}^{i}\left(t, x, y, \tilde{K}^{i}, \tilde{C}^{i}\right)$ where $\tilde{Z}^{i}$ is a intuitionistic fuzzy solution. Let $\tilde{K}^{i}[\alpha]=\prod_{j=1}^{n} \tilde{K}_{j}^{i}[\alpha]$ and $\tilde{C}^{i}[\alpha]=\prod_{r=1}^{m} \tilde{C}_{r}^{i}[\alpha]$.

Of cours, we mean that the $G$ in Eq. $(3.2)$ is not defined in terms of a series. That is, there are no Fourier series used to define $G$. Since in this paper we will interested for $G$ with intuitionistic fuzzy parameters we do not wish to consider Fourier series in intuitionistic fuzzy sets concept. We need the solution $G$ to be fairly simple. So, we also assume that Bessel functions and Legendre functions are not used in $G$. 


\section{The VARiational iteration method}

To illustrate the basic idea of the VIM we consider the following PDE model

$$
L_{t} U+L_{x} U+L_{y} U+N U=F(t, x, y, k)
$$

where $L_{t}, L_{x}$ and $L_{y}$ are linear operators of $\mathrm{t}, \mathrm{x}$ and $\mathrm{y}$, respectively, and $\mathrm{N}$ is a nonlinear operator, also $F(t, x, y, k)$ is the source non-homogeneous term. According to the VIM [11, we can express the following correction function (4.1) in $\mathrm{t}, \mathrm{x}$ and $\mathrm{y}$ directions can be written as

$$
U_{n+1}(t, x, y)=U_{n}(t, x, y)+\int_{0}^{t} \lambda\left\{L_{s} U_{n}+\left(L_{x}+L_{y}+N\right) \widetilde{U}_{n}-F(s, x, y, k)\right\} d s
$$

where $\lambda$ is general Lagrange multiplier [8, which can be identified optimally via the variational theory [1], and $\widetilde{U}_{n}$ is a restricted variation which means $\delta \widetilde{U}_{n}=0$. It is required first to determine the Lagrange multipliers $\lambda$ that will be identified optimally via integration by parts. The approximations $U_{n+1}, n \geq 0$, of the solution $U(t, x, y)$ will immediately follow upon using any selective function $U_{0}$.

The initial values $U(0, x, y)$ is usually used for the selected zeroth approximations $U_{0}$. With the Lagrange multiplies $\lambda$ determined, then several approximation $u_{i}(t, x, y), i \geq 0$ can be determined. Consequently, the solution is given as

$$
U(t, x, y)=\lim _{n \rightarrow \infty} U_{n}(t, x, y)
$$

According to the VIM, we construct a correction functional for 3.2 in t-direction as follows

$$
U_{n+1}(t, x, y)=U_{n}(t, x, y)+\int_{0}^{t} \lambda(s)\left\{\left(U_{n}\right)_{s}+P(x)\left(\widetilde{U}_{n}\right)_{x x}+Q(y)\left(\widetilde{U}_{n}\right)_{y y}-F(s, x, y, k)\right\} d s
$$

where $n \geq 0$ and $\lambda$ is a Lagrange multiplier. We now determine the Lagrange multiplier

$$
\delta U_{n+1}(t, x, y)=\delta U_{n}(t, x, y)+\delta \int_{0}^{t} \lambda(s)\left\{\left(U_{n}\right)_{s}+P(x)\left(\widetilde{U}_{n}\right)_{x x}+Q(y)\left(\widetilde{U}_{n}\right)_{y y}-F(s, x, y, k)\right\} d s
$$

Therefore, the stationary conditions are : $\lambda^{\prime}(s)=0$ and $1+\left.\lambda(s)\right|_{s=t}=0$.

So, the Lagrange multiplier is $\lambda=-1$. Submitting the results into 4.4 leads to the following iteration formula

$$
U_{n+1}(t, x, y)=U_{n}(t, x, y)-\int_{0}^{t}\left\{\left(U_{n}\right)_{s}+P(x)\left(\widetilde{U}_{n}\right)_{x x}+Q(y)\left(\widetilde{U}_{n}\right)_{y y}-F(s, x, y, k)\right\} d s
$$

Iteration formula start with initial approximation, for example $U_{0}(t, x, y)=U(0, x, y)$. Also the VIM used for system of linear and nonlinear partial differential equation [8] which handled in obtain the solution.

\section{Solution CONCEPT}

The united extension of $f$ for $\alpha \in[0,1]$ is $\Omega[\alpha]=\left(\Omega^{+}[\alpha], \Omega^{-}[\alpha]\right)$

$$
\Omega^{+}[\alpha]=\left\{y \mid y=f(x), x \in A^{+}[\alpha]\right\} \text { and } \Omega^{-}[\alpha]=\left\{y \mid y=f(x), x \in A^{-}[\alpha]\right\}
$$

Define $\tilde{C}^{i}$, a intuitionistic fuzzy subset of the real numbers, by its membership function

$$
\mu_{\tilde{C}^{i}}(y)=\sup \left\{\alpha \mid y \in \Omega^{+}[\alpha]\right\}, \quad \nu_{\tilde{C}^{i}}(y)=1-\sup \left\{\alpha \mid y \in \Omega^{-}[\alpha]\right\}
$$

Theorem 5.1. If $f$ is continuous, then $\tilde{C}^{i}[\alpha]=\Omega[\alpha]$ where $\left.\left.\alpha \in\right] 0,1\right]$

Proof. All we need to show is that $\tilde{C}^{i}[\alpha]=\Omega[\alpha]$ since,

- Let $y \in \Omega[\alpha]$. Then $\mu_{\tilde{C}^{i}}(y) \geq \alpha$ and $\nu_{\tilde{C}^{i}}(y) \leq 1-\alpha$ so that $y \in \tilde{C}^{i}[\alpha]$. 
- Let $y \in \tilde{C}^{i}[\alpha]$ so that $\mu_{\tilde{C}^{i}}(y)=\alpha_{0} \geq \alpha$ and $\nu_{\tilde{C}^{i}}(y)=1-\alpha_{0} \leq 1-\alpha$. Choose $\left\{\alpha_{n}\right\}$ such that $0 \leq \alpha_{n} \uparrow \alpha_{0}$ and $\alpha_{n}<\alpha_{0}$. We know that $y \in \Omega\left[\alpha_{n}\right]$ from the definition of supremum used to define $\tilde{C}^{i}$. Choose $z_{n} \in \tilde{A}^{i}\left[\alpha_{n}\right]$ so that $f\left(z_{n}\right)=y$. Since $z_{n} \in \tilde{A}^{i}[0]$, which is compact, there is a convergent subsequence $z_{n_{k}} \rightarrow z^{*} \in \tilde{A}^{i}\left[\alpha_{0}\right]$. Hence $f\left(z_{n_{k}}\right) \rightarrow f\left(z^{*}\right)=y$ and $y \in \Omega\left[\alpha_{0}\right]$. Therefore, $y \in \Omega[\alpha]$ because $\alpha \leq \alpha_{0}$.

Using Theorem 5.1 we may now discuss the first solution.

5.1. The first solution. They define for all $t, x, y$ and $\alpha$,

$$
\begin{aligned}
\tilde{Z}^{i}(t, x, y)[\alpha] & =\left\{\left[z_{1}^{+}(t, x, y, \alpha), z_{2}^{+}(t, x, y, \alpha)\right],\left[z_{1}^{-}(t, x, y, \alpha), z_{2}^{-}(t, x, y, \alpha)\right]\right\} \text { and } \\
\tilde{F}^{i}\left(t, x, y, \tilde{K}^{i}\right)[\alpha] & =\left\{\left[F_{1}^{+}(t, x, y, \alpha), F_{2}^{+}(t, x, y, \alpha)\right],\left[F_{1}^{-}(t, x, y, \alpha), F_{2}^{-}(t, x, y, \alpha)\right]\right\}
\end{aligned}
$$

Let $W=K^{+}[\alpha] \times C^{+}[\alpha]$ and $P=K^{-}[\alpha] \times C^{-}[\alpha]$. By definition

$$
\begin{aligned}
& z_{1}^{+}(t, x, y, \alpha)=\min \{G(t, x, y, k, c):(k, c) \in W\} \\
& z_{2}^{+}(t, x, y, \alpha)=\max \{G(t, x, k, y, c):(k, c) \in W\} \\
& z_{1}^{-}(t, x, y, \alpha)=\min \{G(t, x, y, k, c):(k, c) \in P\} \\
& z_{2}^{-}(t, x, y, \alpha)=\max \{G(t, x, k, y, c):(k, c) \in P\}
\end{aligned}
$$

$\forall(t, x, y) \in \prod_{j=1}^{3} I_{j} \quad$ and $\quad \alpha \in[0,1]$, and

$$
\begin{aligned}
& F_{1}^{+}(t, x, y, \alpha)=\min \left\{F(t, x, y, k): k \in K^{+}[\alpha]\right\} \\
& F_{2}^{+}(t, x, y, \alpha)=\max \left\{F(t, x, y, k): k \in K^{+}[\alpha]\right\} \\
& F_{1}^{-}(t, x, y, \alpha)=\min \left\{F(t, x, y, k): k \in K^{-}[\alpha]\right\} \\
& F_{2}^{-}(t, x, y, \alpha)=\max \left\{F(t, x, y, k): k \in K^{-}[\alpha]\right\}
\end{aligned}
$$

$\forall(t, x, y) \in \prod_{j=1}^{3} I_{j}$ and $\alpha \in[0,1]$

Assume that $P(x)>0, Q(y)>0$ and the $z_{i}^{\triangle}(t, x, y, \alpha) i=1,2$ and $\triangle \in\{+,-\}$, has continuous partial derivatives so $\left(z_{i}^{+}\right)_{t}+P(x)\left(z_{i}^{+}\right)_{x x}+Q(y)\left(z_{i}^{+}\right)_{y y}$ and $\left(z_{i}^{-}\right)_{t}+P(x)\left(z_{i}^{-}\right)_{x x}+Q(y)\left(z_{i}^{-}\right)_{y y}$ are continuous for all $(t, x, y) \in \prod_{j=1}^{3} I_{j}$ and all $\alpha \in[0,1]$. Define

$$
\begin{aligned}
\Gamma(t, x, y, \alpha)=\left\{\left[\left(z_{1}^{+}\right)_{t}+P(x)\left(z_{1}^{+}\right)_{x x}+Q(y)\left(z_{1}^{+}\right)_{y y},\left(z_{2}^{+}\right)_{t}+P(x)\left(z_{2}^{+}\right)_{x x}+Q(y)\left(z_{2}^{+}\right)_{y y}\right]\right. \\
{\left.\left[\left(z_{1}^{-}\right)_{t}+P(x)\left(z_{1}^{-}\right)_{x x}+Q(y)\left(z_{1}^{-}\right)_{y y},\left(z_{2}^{-}\right)_{t}+P(x)\left(z_{2}^{-}\right)_{x x}+Q(y)\left(z_{2}^{-}\right)_{y y}\right]\right\} }
\end{aligned}
$$

for all $(t, x, y) \in \prod_{j=1}^{3} I_{j}$ and all $\alpha$.

If, for each fixed $t, x, y \in \prod_{j=1}^{3} I_{j}, \Gamma(t, x, y, \alpha)$ defines the $\alpha$-cut of a intuitionistic fuzzy number, then will be said that $\tilde{Z}^{i}(t, x, y)$ is differentiable and is written

$$
\tilde{Z}_{t}^{i}[\alpha]+P(x) \tilde{Z}_{x x}^{i}[\alpha]+Q(y) \tilde{Z}_{y y}^{i}[\alpha]=\Gamma(t, x, y, \alpha)
$$


for all $(t, x, y) \in \prod_{j=1}^{3} I_{j}$ and all $\alpha$

Sufficient conditions for $\Gamma(t, x, y, \alpha)$ to define $\alpha$-cut of a intuitionistic fuzzy number are [10] :

(i) $\left(z_{1}^{+}\right)_{t}(t, x, y, \alpha)+P(x)\left(z_{1}^{+}\right)_{x x}(t, x, y, \alpha)+Q(y)\left(z_{1}^{+}\right)_{y y}(t, x, y, \alpha)$ and

$$
\left(z_{2}^{-}\right)_{t}(t, x, y, \alpha)+P(x)\left(z_{2}^{-}\right)_{x x}(t, x, y, \alpha)+Q(y)\left(z_{2}^{-}\right)_{y y}(t, x, y, \alpha)
$$

are an increasing function of $\alpha$ for each $(t, x, y) \in \prod_{j=1}^{3} I_{j}$

(ii) $\left(z_{2}^{+}\right)_{t}(t, x, y, \alpha)+P(x)\left(z_{2}^{+}\right)_{x x}(t, x, y, \alpha)+Q(y)\left(z_{2}^{+}\right)_{y y}(t, x, y, \alpha) \quad$ and

$$
\left(z_{1}^{-}\right)_{t}(t, x, y, \alpha)+P(x)\left(z_{1}^{-}\right)_{x x}(t, x, y, \alpha)+Q(y)\left(z_{1}^{-}\right)_{y y}(t, x, y, \alpha)
$$

are an decreasing function of $\alpha$ for each $(t, x, y) \in \prod_{j=1}^{3} I_{j}$ and

(iii) for $(t, x, y) \in \prod_{j=1}^{3} I_{j}$

$$
\begin{aligned}
\left(z_{1}^{+}\right)_{t}(t, x, y, 1)+ & P(x)\left(z_{1}^{+}\right)_{x x}(t, x, y, 1)+Q(y)\left(z_{1}^{+}\right)_{y y}(t, x, y, 1) \\
\leq & \left(z_{2}^{+}\right)_{t}(t, x, y, 1)+P(x)\left(z_{2}^{+}\right)_{x x}(t, x, y, 1)+Q(y)\left(z_{2}^{+}\right)_{y y}(t, x, y, 1)
\end{aligned}
$$

(iv) for $(t, x, y) \in \prod_{j=1}^{3} I_{j}$

$$
\begin{aligned}
\left(z_{1}^{-}\right)_{t}(t, x, y, 0)+ & P(x)\left(z_{1}^{-}\right)_{x x}(t, x, y, 0)+Q(y)\left(z_{1}^{-}\right)_{y y}(t, x, y, 0) \\
& \leq\left(z_{2}^{-}\right)_{t}(t, x, y, 0)+P(x)\left(z_{2}^{-}\right)_{x x}(t, x, y, 0)+Q(y)\left(z_{2}^{-}\right)_{y y}(t, x, y, 0)
\end{aligned}
$$

Now we assume that the $z_{i}^{\triangle}(t, x, y, \alpha)$ for $\triangle \in\{+,-\}$ has continuous partial derivatives, so $\left(z_{i}^{+}\right)_{t}+P(x)\left(z_{i}^{+}\right)_{x x}+$ $Q(y)\left(z_{i}^{+}\right)_{y y}$ and $\left(z_{i}^{-}\right)_{t}+P(x)\left(z_{i}^{-}\right)_{x x}+Q(y)\left(z_{i}^{-}\right)_{y y}$ are continuous on $\prod_{j=1}^{3} I_{j} \times[0,1] i=1,2$. Hence, if conditions $(i)-(i v)$ above hold, $\tilde{Z}^{i}(t, x, y)$ is differentiable.

For $\tilde{Z}^{i}(t, x, y)$ to be a first solution $(1-S)$ of the intuitionistic fuzzy heat-like equation we need

(a) $\tilde{Z}^{i}(t, x, y)$ differentiable

(b) 3.5 holds for $\tilde{U}^{i}(t, x, y)=\tilde{Z}^{i}(t, x, y)$,

(c) $\tilde{Z}^{i}(t, x, y)$ satisfies the initial and boundary conditions. Since no exist specified any particular initial and boundary conditions, then only is checked if $(3.5)$ hold.

$\tilde{Z}^{i}(t, x, y)$ is a $(1-S)$ (without the initial and boundary conditions) if $\tilde{Z}^{i}(t, x, y)$ is differentiable and $\left(\tilde{Z}^{i}\right)_{t}+$ $P(x)\left(\tilde{Z}^{i}\right)_{x x}+Q(y)\left(\tilde{Z}^{i}\right)_{y y}=\tilde{F}^{i}\left(t, x, y, \tilde{K}^{i}\right)$ or the following equations must hold

$$
\begin{aligned}
& \left(z_{1}^{+}\right)_{t}+P(x)\left(z_{1}^{+}\right)_{x x}+Q(y)\left(z_{1}^{+}\right)_{y y}=F_{1}^{+}(t, x, y, \alpha) \\
& \left(z_{2}^{+}\right)_{t}+P(x)\left(z_{2}^{+}\right)_{x x}+Q(y)\left(z_{2}^{+}\right)_{y y}=F_{2}^{+}(t, x, y, \alpha) \\
& \left(z_{1}^{-}\right)_{t}+P(x)\left(z_{1}^{-}\right)_{x x}+Q(y)\left(z_{1}^{-}\right)_{y y}=F_{1}^{-}(t, x, y, \alpha) \\
& \left(z_{2}^{-}\right)_{t}+P(x)\left(z_{2}^{-}\right)_{x x}+Q(y)\left(z_{2}^{-}\right)_{y y}=F_{2}^{-}(t, x, y, \alpha)
\end{aligned}
$$

for all $(t, x, y) \in \prod_{j=1}^{3} I_{j}$ and $\alpha \in[0,1]$.

Now we will present a sufficient condition for the $(1-S)$ to exist. Since there are such a variety of possible initial and boundary conditions, so we will omit them from the following theorem. One must separately check out the initial and boundary conditions. So, we will omit the constants $c_{i}, 1 \leq i \leq m$, from the problem. Therefore, (3.4) becomes $U(t, x, y)=G(t, x, y, k$,$) , so \tilde{Z}^{i}(t, x, y)=\tilde{G}^{i}\left(t, x, y, \tilde{K}^{i}\right)$.

Theorem 5.2. Assume $\tilde{Z}^{i}(t, x, y)$ is differentiable.

(a) If

$$
P(x)>0 \text { and } Q(y)>0 \quad(x, y) \in I_{2} \times I_{3}
$$


and if

$$
\frac{\partial G}{\partial k_{j}} \frac{\partial F}{\partial k_{j}}>0 \text { for } j=1,2, \ldots, n
$$

Then $\tilde{Z}^{i}(t, x, y)$ is $(1-S)$

(b) If relations (5.15) does not hold or relation (5.16) does not hold for some $j$, then $\tilde{Z}^{i}(t, x, y)$ is not a (1-S).

Proof. (a) For simplicity assume $k_{j}=k$ and $\frac{\partial G}{\partial k}<0, \frac{\partial F}{\partial k}<0$. The proof for $\frac{\partial G}{\partial k}>0, \frac{\partial F}{\partial k}>0$, is similar and omitted.

Since $\frac{\partial G}{\partial k}<0$ then from $(5.1)$ and $(5.2)$ we have

$$
\begin{array}{ll}
z_{1}^{+}(t, x, y, \alpha)=G\left(t, x, y, k_{2}^{+}(\alpha)\right), & z_{2}^{+}(t, x, y, \alpha)=G\left(t, x, y, k_{1}^{+}(\alpha)\right) \\
z_{1}^{-}(t, x, y, \alpha)=G\left(t, x, y, k_{2}^{-}(\alpha)\right), & z_{2}^{-}(t, x, y, \alpha)=G\left(t, x, y, k_{1}^{-}(\alpha)\right)
\end{array}
$$

from 5.5, 5.6 and $\frac{\partial F}{\partial k}<0$ we have

$$
\begin{aligned}
& F_{1}^{+}(t, x, y, \alpha)=F\left(t, x, y, k_{2}^{+}(\alpha)\right), \quad F_{2}^{+}(t, x, y, \alpha)=F\left(t, x, y, k_{1}^{+}(\alpha)\right) \\
& F_{1}^{-}(t, x, y, \alpha)=F\left(t, x, y, k_{2}^{-}(\alpha)\right), \quad F_{2}^{-}(t, x, y, \alpha)=F\left(t, x, y, k_{1}^{-}(\alpha)\right)
\end{aligned}
$$

for all $\alpha \in[0,1]$ where $\tilde{K}^{i}[\alpha]=\left(\left[k_{1}^{+}(\alpha), k_{2}^{+}(\alpha)\right],\left[k_{1}^{-}(\alpha), k_{2}^{-}(\alpha)\right]\right)$.

Now $G(t, x, y, k)$ solves 3.2 , which means

$$
G_{t}+P(x) G_{x x}+Q(y) G_{y y}=F(t, x, y, k)
$$

for all $(t, x, y) \in \prod_{j=1}^{3} I_{j}, k \in J$.

Suppose $\bar{Z}(t, x, y)$ is differentiable and $P(x)>0$ and $Q(y)>0$ so

$$
\begin{aligned}
& \partial_{t} z_{1}^{+}(t, x, y, \alpha)+P(x) \partial_{x x} z_{1}^{+}(t, x, y, \alpha)+Q(y) \partial_{y y} z_{1}^{+}(t, x, y, \alpha)=F_{1}^{+}(t, x, y, \alpha) \\
& \partial_{t} z_{2}^{+}(t, x, y, \alpha)+P(x) \partial_{x x} z_{2}^{+}(t, x, y, \alpha)+Q(y) \partial_{y y} z_{2}^{+}(t, x, y, \alpha)=F_{2}^{+}(t, x, y, \alpha) \\
& \partial_{t} z_{1}^{-}(t, x, y, \alpha)+P(x) \partial_{x x} z_{1}^{-}(t, x, y, \alpha)+Q(y) \partial_{y y} z_{1}^{-}(t, x, y, \alpha)=F_{1}^{-}(t, x, y, \alpha) \\
& \partial_{t} z_{2}^{-}(t, x, y, \alpha)+P(x) \partial_{x x} z_{2}^{-}(t, x, y, \alpha)+Q(y) \partial_{y y} z_{2}^{-}(t, x, y, \alpha)=F_{2}^{-}(t, x, y, \alpha)
\end{aligned}
$$

for all $(t, x, y) \in \prod_{j=1}^{3} I_{j}$ and $\alpha \in[0,1]$

Hence, 5.11, 5.12), 5.13, 5.14 hold and $\tilde{Z}^{i}(t, x, y)$ is a $(1-S)$.

(b) Now consider the situation where (5.15) or (5.16) does not hold.

Let us only look at one case where $\frac{\partial F}{\partial k}<0\left(\right.$ assume $\frac{\partial G}{\partial k}>0, P(x)>0$ and $\left.Q(y)>0\right)$. Then we have

$$
\begin{aligned}
z_{1}^{+}(t, x, y, \alpha)=G\left(t, x, y, k_{1}^{+}(\alpha)\right) & z_{2}^{+}(t, x, y, \alpha)=G\left(t, x, y, k_{2}^{+}(\alpha)\right) \\
z_{1}^{-}(t, x, y, \alpha)=G\left(t, x, y, k_{1}^{-}(\alpha)\right) & z_{2}^{-}(t, x, y, \alpha)=G\left(t, x, y, k_{2}^{-}(\alpha)\right) \\
F_{1}^{+}(t, x, y, \alpha)=F\left(t, x, y, k_{2}^{+}(\alpha)\right) & F_{2}^{+}(t, x, y, \alpha)=F\left(t, x, y, k_{1}^{+}(\alpha)\right) \\
F_{1}^{-}(t, x, y, \alpha)=F\left(t, x, y, k_{2}^{-}(\alpha)\right) & F_{2}^{-}(t, x, y, \alpha)=F\left(t, x, y, k_{1}^{-}(\alpha)\right)
\end{aligned}
$$


then we have

$$
\begin{aligned}
\partial_{t} z_{1}^{+}(t, x, y, \alpha)+P(x) \partial_{x x} z_{1}^{+}(t, x, y, \alpha)+Q(y) \partial_{y y} z_{1}^{+}(t, x, y, \alpha) & =F_{1}^{+}(t, x, y, \alpha) \\
\partial_{t} z_{2}^{+}(t, x, y, \alpha)+P(x) \partial_{x x} z_{2}^{+}(t, x, \alpha)+Q(y) \partial_{y y} z_{2}^{+}(t, x, y, \alpha) & =F_{2}^{+}(t, x, y, \alpha) \\
\partial_{t} z_{1}^{-}(t, x, y, \alpha)+P(x) \partial_{x x} z_{1}^{-}(t, x, y, \alpha)+Q(y) \partial_{y y} z_{1}^{-}(t, x, y, \alpha) & =F_{1}^{-}(t, x, y, \alpha) \\
\partial_{t} z_{2}^{-}(t, x, y, \alpha)+P(x) \partial_{x x} z_{2}^{-}(t, x, \alpha)+Q(y) \partial_{y y} z_{2}^{-}(t, x, y, \alpha) & =F_{2}^{-}(t, x, y, \alpha)
\end{aligned}
$$

which are not true. because

$$
\begin{gathered}
G_{t}\left(t, x, y, k_{1}^{+}(\alpha)\right)+P(x) G_{x x}\left(t, x, y, k_{1}^{+}(\alpha)\right)+Q(y) G_{y y}\left(t, x, y, k_{1}^{+}(\alpha)\right)=F\left(t, x, y, k_{2}^{+}(\alpha)\right) \\
G_{t}\left(t, x, y, k_{2}^{+}(\alpha)\right)+P(x) G_{x x}\left(t, x, y, k_{2}^{+}(\alpha)\right)+Q(y) G_{y y}\left(t, x, k_{2}^{+}(\alpha)\right)=F\left(t, x, y, k_{1}^{+}(\alpha)\right) \\
G_{t}\left(t, x, y, k_{1}^{-}(\alpha)\right)+P(x) G_{x x}\left(t, x, y, k_{1}^{-}(\alpha)\right)+Q(y) G_{y y}\left(t, x, y, k_{1}^{-}(\alpha)\right)=F\left(t, x, y, k_{2}^{-}(\alpha)\right) \\
G_{t}\left(t, x, y, k_{2}^{-}(\alpha)\right)+P(x) G_{x x}\left(t, x, y, k_{2}^{-}(\alpha)\right)+Q(y) G_{y y}\left(t, x, k_{2}^{-}(\alpha)\right)=F\left(t, x, y, k_{1}^{-}(\alpha)\right)
\end{gathered}
$$

Therefore, if $\tilde{Z}^{i}(t, x, y)$ is a $(1-S)$ and it satisfies the initial and boundary conditions we will say that $\tilde{Z}^{i}(t, x, y)$ is a $(1-S)$ satisfying the initial and boundary conditions. If $\tilde{Z}^{i}(t, x, y)$ is not a $(1-S)$, then we will consider the second solution $(2-S)$.

5.2. The second solution. Now let us define the second solution $(2-S)$. Let

$$
\tilde{U}^{i}(t, x, y)[\alpha]=\left\{\left[u_{1}^{+}(t, x, y, \alpha), u_{2}^{+}(t, x, y, \alpha)\right],\left[u_{1}^{-}(t, x, y, \alpha), u_{2}^{-}(t, x, y, \alpha)\right]\right\}
$$

For example suppose $P(x)<0$ and $Q(y)>0$, so consider the system of heat-like equations

$$
\begin{aligned}
& \left(u_{1}^{+}\right)_{t}+P(x)\left(u_{2}^{+}\right)_{x x}+Q(y)\left(u_{1}^{+}\right)_{y y}=F_{1}^{+}(t, x, y, \alpha) \\
& \left(u_{2}^{+}\right)_{t}+P(x)\left(u_{1}^{+}\right)_{x x}+Q(y)\left(u_{2}^{+}\right)_{y y}=F_{2}^{+}(t, x, y, \alpha) \\
& \left(u_{1}^{-}\right)_{t}+P(x)\left(u_{2}^{-}\right)_{x x}+Q(y)\left(u_{1}^{-}\right)_{y y}=F_{1}^{-}(t, x, y, \alpha) \\
& \left(u_{2}^{-}\right)_{t}+P(x)\left(u_{1}^{-}\right)_{x x}+Q(y)\left(u_{2}^{-}\right)_{y y}=F_{2}^{-}(t, x, y, \alpha)
\end{aligned}
$$

or if $P(x)>0, Q(y)>0, \frac{\partial F}{\partial k}>0, \frac{\partial G}{\partial k}<0$,

$$
\begin{aligned}
& \left(u_{1}^{+}\right)_{t}+P(x)\left(u_{1}^{+}\right)_{x x}+Q(y)\left(u_{1}^{+}\right)_{y y}=F_{1}^{+}(t, x, y, \alpha) \\
& \left.\left(u_{2}^{+}\right)_{t}+P(x)\left(u_{2}^{+}\right)_{x x}+Q(y)\left(u_{2}^{+}\right)_{y y}=F_{2}^{+}(t, x, y, \alpha)\right) \\
& \left(u_{1}^{-}\right)_{t}+P(x)\left(u_{1}^{-}\right)_{x x}+Q(y)\left(u_{1}^{-}\right)_{y y}=F_{1}^{-}(t, x, y, \alpha) \\
& \left.\left(u_{2}^{-}\right)_{t}+P(x)\left(u_{2}^{-}\right)_{x x}+Q(y)\left(u_{2}^{-}\right)_{y y}=F_{2}^{-}(t, x, y, \alpha)\right)
\end{aligned}
$$

for all $(t, x, y) \in \prod_{j=1}^{3} I_{j}$ and $\alpha \in[0,1]$. We append to 5.20 and $\sqrt{5.21}$ any initial and boundary conditions. For example, if it was $\tilde{U}^{i}(0, x, y)=\tilde{C}^{i}$ then we add

$$
\begin{aligned}
& u_{1}^{+}(0, x, y, \alpha)=c_{1}^{+}(\alpha) \quad u_{1}^{-}(0, x, y, \alpha)=c_{1}^{-}(\alpha) \\
& u_{2}^{+}(0, x, y, \alpha)=c_{2}^{+}(\alpha) \quad u_{2}^{-}(0, x, y, \alpha)=c_{2}^{-}(\alpha)
\end{aligned}
$$


where $\tilde{C}^{i}[\alpha]=\left\{\left[c_{1}^{+}(\alpha), c_{2}^{+}(\alpha)\right],\left[c_{1}^{-}(\alpha), c_{2}^{-}(\alpha)\right]\right\}$.

Let $u_{i}^{\triangle}(t, x, y, \alpha)$ for $i=1,2$ and $\triangle \in\{+,-\}$ solve 5.20 and 5.21 plus initial and boundary conditions.

If

$$
\left[u_{1}^{+}(t, x, y, \alpha), u_{2}^{+}(t, x, y, \alpha)\right] \text { and }\left[u_{1}^{-}(t, x, y, \alpha), u_{2}^{-}(t, x, y, \alpha)\right]
$$

defines the $\alpha$-cut of a intuitionistic fuzzy number, for all $(t, x, y) \in \prod_{j=1}^{3} I_{j}$, then $\tilde{U}^{i}(t, x, y)$ is the $(2-S)$.

We will say that derivative condition holds for intuitionistic fuzzy heat-like equation when Eqs. (5.15) and (5.16) are true.

Theorem 5.3. (1) If $(1-S)=\tilde{Z}^{i}(t, x, y)$, then $(2-S)=\tilde{Z}^{i}(t, x, y)$

(2) If $(2-S)=\tilde{Z}^{i}(t, x, y)$ and the derivative condition holds, then $(1-S)=\tilde{U}^{i}(t, x, y)$

Proof. (1) Follows from the definition of $(1-S)$ and $(2-S)$.

(2) If $(2-S)=\tilde{U}^{i}(t, x, y)$ then the derivative exists and since the derivative condition holds, therefore, Eqs. following holds

$$
\begin{aligned}
& \left(u_{1}^{+}\right)_{t}+P(x)\left(u_{1}^{+}\right)_{x x}+Q(y)\left(u_{1}^{+}\right)_{y y}=F_{1}^{+}(t, x, y, \alpha) \\
& \left(u_{2}^{+}\right)_{t}+P(x)\left(u_{2}^{+}\right)_{x x}+Q(y)\left(u_{2}^{+}\right)_{y y}=F_{2}^{+}(t, x, y, \alpha) \\
& \left(u_{1}^{-}\right)_{t}+P(x)\left(u_{1}^{-}\right)_{x x}+Q(y)\left(u_{1}^{-}\right)_{y y}=F_{1}^{-}(t, x, y, \alpha) \\
& \left(u_{2}^{-}\right)_{t}+P(x)\left(u_{2}^{-}\right)_{x x}+Q(y)\left(u_{2}^{-}\right)_{y y}=F_{2}^{-}(t, x, y, \alpha)
\end{aligned}
$$

Also suppose one $k_{j}=k, \frac{\partial G}{\partial k}<0$ and $\frac{\partial F}{\partial k}<0$ (the other cases are similar and are omitted). We see

$$
\begin{aligned}
& z_{1}^{+}(t, x, y, \alpha)=G\left(t, x, y, k_{2}^{+}(\alpha)\right) \quad z_{2}^{+}(t, x, y, \alpha)=G\left(t, x, y, k_{1}^{+}(\alpha)\right) \\
& z_{1}^{-}(t, x, y, \alpha)=G\left(t, x, y, k_{2}^{-}(\alpha)\right) \quad z_{2}^{-}(t, x, y, \alpha)=G\left(t, x, y, k_{1}^{-}(\alpha)\right) \\
& F_{1}^{+}(t, x, y, \alpha)=F\left(t, x, y, k_{2}^{+}(\alpha)\right), \quad F_{2}^{+}(t, x, y, \alpha)=F\left(t, x, y, k_{1}^{+}(\alpha)\right) \\
& F_{1}^{-}(t, x, y, \alpha)=F\left(t, x, y, k_{2}^{-}(\alpha)\right), \quad F_{2}^{-}(t, x, y, \alpha)=F\left(t, x, y, k_{1}^{-}(\alpha)\right)
\end{aligned}
$$

Now look at Eqs. (5.11), (5.12), 55.13, (5.14) also Eqs. (5.1), 5.2), implies that

$$
\begin{aligned}
& u_{1}^{+}(t, x, y, \alpha)=G\left(\left(t, x, y, k_{2}^{+}(\alpha)\right)=z_{1}^{+}(t, x, y, \alpha)\right. \\
& u_{2}^{+}(t, x, y, \alpha)=G\left(t, x, y, k_{1}^{+}(\alpha)\right)=z_{2}^{+}(t, x, y, \alpha) \\
& u_{1}^{-}(t, x, y, \alpha)=G\left(\left(t, x, y, k_{2}^{-}(\alpha)\right)=z_{1}^{-}(t, x, y, \alpha)\right. \\
& u_{2}^{-}(t, x, y, \alpha)=G\left(t, x, y, k_{1}^{-}(\alpha)\right)=z_{2}^{-}(t, x, y, \alpha)
\end{aligned}
$$

Therefore $(1-S)=\tilde{U}^{i}(t, x, y)$

Lemma 5.4. Consider (3.1) suppose $\tilde{Z}^{i}(t, x)$ is differentiable.

(a) If

$$
P(x)>0 \quad x \in I_{2}
$$

and if

$$
\frac{\partial G}{\partial k_{j}} \frac{\partial F}{\partial k_{j}}>0 \text { for } j=1,2, \ldots, n
$$


Then $(1-S)=\tilde{Z}^{i}(t, x)$

(b) If relations (5.31) does not hold or relation (5.32) does not hold for some $j$, then $\tilde{Z}^{i}(t, x)$ is not a $(1-S)$.

Proof. It is similar to theorem 5.2

\section{Examples}

We consider the following examples $([5])$ and we added intuitionistic fuzzy parameters to these reference.

Example 6.1. We first consider the one-dimensional heat-like equation with variable coefficients as

$$
U_{t}+\frac{1}{2} x^{2} U_{x x}=k
$$

with the initial condition

$$
U(0, x)=c x^{2}
$$

where $t \in\left(0, M_{1}\right], x \in\left(0, M_{2}\right], k \in[0, J]$ is constant.

According to the VIM, a correct functional for 6.1 from (4.5) can be constructed as follows

$$
U_{n+1}(t, x)=U_{n}(t, x)-\int_{0}^{t}\left\{\left(U_{n}\right)_{s}(s, x)+\frac{1}{2} x^{2}\left(\widetilde{U}_{n}\right)_{x x}(s, x)-F(s, x, k)\right\} d s
$$

Beginning with an initial approximation $U_{0}(t, x)=U(0, x)=c x^{2}$, we can obtain the following successive approximations $U_{1}(t, x)=c x^{2}(1-t)+k t$

$U_{2}(t, x)=c x^{2}\left(1-t+\frac{t^{2}}{2 !}\right)+k t$

$U_{3}(t, x)=c x^{2}\left(1-t+\frac{t^{2}}{2 !}-\frac{t^{3}}{3 !}\right)+k t$

and $U_{n}(t, x)=c x^{2}\left(1-t+\frac{t^{2}}{2 !}-\frac{t^{3}}{3 !}+\ldots .+(-1)^{n} \frac{t^{n}}{n !}\right)+k t, n \geq 1$

The VIM admits the use of $U(t, x)=\lim _{n \rightarrow \infty} U_{n}(t, x)$, which gives the exact solution

$$
U(t, x)=c x^{2} \exp (-t)+k t
$$

and extension for intuitionistic fuzzy sets of $F(t, x, k)$ and $G(t, x, k, c)=c x^{2} \exp (-t)+k t$. Clearly $\tilde{F}^{i}\left(t, x, \tilde{K}^{i}\right)=\tilde{K}^{i}$ so that

$$
\begin{aligned}
& F_{1}^{+}(t, x, \alpha)=k_{1}^{+}(\alpha), \quad F_{2}^{+}(t, x, \alpha)=k_{2}^{+}(\alpha) \\
& F_{1}^{-}(t, x, \alpha)=k_{1}^{-}(\alpha), \quad F_{2}^{-}(t, x, \alpha)=k_{2}^{-}(\alpha)
\end{aligned}
$$

Also $\tilde{G}^{i}\left(t, x, \tilde{K}^{i}, \tilde{C}^{i}\right)=\tilde{C}^{i} x^{2} \exp (-t)+\tilde{K}^{i} t$, therefore for $i=1,2$

$$
\begin{gathered}
z_{i}^{+}(t, x, \alpha)=c_{i}^{+}(\alpha) x^{2} \exp (-t)+k_{i}^{+}(\alpha) t \\
z_{i}^{-}(t, x, \alpha)=c_{i}^{-}(\alpha) x^{2} \exp (-t)+k_{i}^{-}(\alpha) t \\
\tilde{K}^{i}[\alpha]=\left(\left[k_{1}^{+}(\alpha), k_{2}^{+}(\alpha)\right],\left[k_{1}^{-}(\alpha), k_{2}^{-}(\alpha)\right]\right) \quad \text { and } \quad \tilde{C}^{i}[\alpha]=\left(\left[c_{1}^{+}(\alpha), c_{2}^{+}(\alpha)\right],\left[c_{1}^{+}(\alpha), c_{2}^{+}(\alpha)\right]\right) .
\end{gathered}
$$

$\tilde{Z}^{i}(t, x)$ is differentiable because

$$
\begin{aligned}
\left(z_{i}^{+}(t, x, \alpha)\right)_{t}+\frac{1}{2} x^{2}\left(z_{i}^{+}(t, x, \alpha)\right)_{x x} & =k_{i}^{+}(\alpha) \\
\left(z_{i}^{-}(t, x, \alpha)\right)_{t}+\frac{1}{2} x^{2}\left(z_{i}^{-}(t, x, \alpha)\right)_{x x} & =k_{i}^{-}(\alpha)
\end{aligned}
$$

for $\mathrm{i}=1,2$ are $\alpha$-cuts of $\tilde{K}^{i}$ i.e $\alpha$-cuts of a intuitionistic fuzzy number. Due to

$$
P(x)>0, \quad \frac{\partial G}{\partial k}>0, \quad \frac{\partial F}{\partial k}>0
$$

That is, $\left(\tilde{Z}^{i}\right)_{t}+\frac{1}{2} x^{2}\left(\tilde{Z}^{i}\right)_{x x}=\tilde{K}^{i}$, a intuitionistic fuzzy number. 
So Lemma 5.4 implies the result that $\tilde{Z}^{i}(t, x)$ is a $(1-S)$. We easily see that

$$
z_{i}^{+}(0, x, \alpha)=c_{i}^{+}(\alpha) x^{2} \quad \text { and } \quad z_{i}^{-}(0, x, \alpha)=c_{i}^{-}(\alpha) x^{2}
$$

for $\mathrm{i}=1,2$, so $\tilde{Z}^{i}(t, x)$ also satisfies the initial condition. The $(1-S)$ that satisfies the initial condition may be written as

$$
\tilde{Z}^{i}(t, x)=\tilde{C}^{i} x^{2} \exp (-t)+\tilde{K}^{i} t \quad \text { for all } \quad(t, x) \in\left(0, M_{1}\right] \times\left(0, M_{2}\right]
$$

Example 6.2. We consider the one-dimensional heat-like model

$$
\begin{aligned}
& U_{t}(t, x)-U_{x x}(t, x)=-K \cos (x) \\
& U(0, x)=C \sin (x)
\end{aligned}
$$

which $x \in\left(0, \frac{\pi}{2}\right), t \in[0, M]$ and the value of parameters $K$ and $C$ are in intervals $[0, J]$ and $[0, L]$, respectively. According to the VIM, a correct functional for Eq. (6.2) from Eq. (4.4) which gives the exact solution

$$
U(t, x)=G(t, x, K, C)=C \exp (-t) \sin (x)+K \cos (x)(\exp (-t)-1)
$$

which is the exact solution. There is no $(1-S)$ because $p(x)=-1<0$ (Lemme5.4).

We proceed to look for a $(2-S)$. We must solve

$$
\begin{aligned}
& \left(u_{1}^{+}\right)_{t}-\left(u_{2}^{+}\right)_{x x}=-k_{2}^{+}(\alpha) \cos (x) \quad\left(u_{2}^{+}\right)_{t}-\left(u_{1}^{+}\right)_{x x}=-k_{1}^{+}(\alpha) \cos (x) \\
& \left(u_{1}^{-}\right)_{t}-\left(u_{2}^{-}\right)_{x x}=-k_{2}^{-}(\alpha) \cos (x) \quad\left(u_{2}^{-}\right)_{t}-\left(u_{1}^{-}\right)_{x x}=-k_{1}^{-}(\alpha) \cos (x)
\end{aligned}
$$

subject to

$$
\begin{array}{ll}
u_{1}^{+}(0, x, \alpha)=c_{1}^{+}(\alpha) \sin (x), & u_{2}^{+}(0, x, \alpha)=c_{2}^{+}(\alpha) \sin (x) \\
u_{1}^{-}(0, x, \alpha)=c_{1}^{-}(\alpha) \sin (x), & u_{2}^{-}(0, x, \alpha)=c_{2}^{-}(\alpha) \sin (x)
\end{array}
$$

If the intervals $\left(\left[u_{1}^{+}(t, x, \alpha), u_{2}^{+}(t, x, \alpha)\right],\left[u_{1}^{-}(t, x, \alpha), u_{2}^{-}(t, x, \alpha)\right]\right)$ define $\alpha$-cuts of a intuitionistic fuzzy number $\tilde{U}^{i}(t, x)$; then $(2-S)=\tilde{U}^{i}(t, x)$. By VIM, the solution is

$$
\begin{array}{ll}
u_{1}^{+}(t, x, \alpha)=c_{1}^{+}(\alpha) \cosh (t) \sin (x)-c_{2}^{+}(\alpha) \sinh (t) \sin (x)+ & \\
& k_{1}^{+}(\alpha) \cos (x)(\cosh (t)-1)-k_{2}^{+}(\alpha) \cos (x) \sinh (t) \\
u_{2}^{+}(t, x, \alpha)=c_{2}^{+}(\alpha) \cosh (t) \sin (x)-c_{1}^{+}(\alpha) \sinh (t) \sin (x)+ & \\
& k_{2}^{+}(\alpha) \cos (x)(\cosh (t)-1)-k_{1}^{+}(\alpha) \cos (x) \sinh (t) \\
u_{1}^{-}(t, x, \alpha)=c_{1}^{-}(\alpha) \cosh (t) \sin (x)-c_{2}^{-}(\alpha) \sinh (t) \sin (x)+ & \\
u_{2}^{-}(t, x, \alpha)=c_{2}^{-}(\alpha) \cosh (t) \sin (x)-c_{1}^{-}(\alpha) \sinh (t) \sin (x)+ & \\
& k_{1}^{-}(\alpha) \cos (x)(\cosh (t)-1)-k_{2}^{-}(\alpha) \cos (x) \sinh (t) \\
& k_{2}^{-}(\alpha) \cos (x)(\cosh (t)-1)-k_{1}^{-}(\alpha) \cos (x) \sinh (t)
\end{array}
$$

Now we show

$$
\left(\left[u_{1}^{+}(t, x, \alpha), u_{2}^{+}(t, x, \alpha)\right],\left[u_{1}^{-}(t, x, \alpha), u_{2}^{-}(t, x, \alpha)\right]\right)
$$

defines $\alpha$-cut of a intuitionistic fuzzy number.

Thus we only need to check if $\frac{\partial u_{1}^{+}}{\partial \alpha}>0, \frac{\partial u_{2}^{+}}{\partial \alpha}<0$ and $\frac{\partial u_{1}^{-}}{\partial \alpha}<0, \frac{\partial u_{2}^{-}}{\partial \alpha}>0$. 
Since $u_{i}^{\triangle}(t, x, \alpha)$ for $\triangle=\{+,-\}$ are continuous and

$$
u_{1}^{+}(t, x, 1)=u_{2}^{+}(t, x, 1) \text { and } u_{1}^{-}(t, x, 0)=u_{2}^{-}(t, x, 0) .
$$

There is a region $\mathfrak{R}$ contained in $(0, M] \times\left(0, \frac{\pi}{2}\right)$ for which the $(2-S)$ exists and $(0, M] \times\left(0, \frac{\pi}{2}\right)-\mathfrak{R}$ there may be no $(2-S)$.

Since $\tilde{K}^{i}$ and $\tilde{C}^{i}$ are triangular intuitionistic fuzzy numbers, hence, we pick simple intuitionistic fuzzy parameter so that $\left(k_{1}^{+}(\alpha)\right)^{\prime},\left(c_{1}^{+}(\alpha)\right)^{\prime},\left(k_{2}^{-}(\alpha)\right)^{\prime}$ and $\left(c_{2}^{-}(\alpha)\right)^{\prime}$ are all positive numbers while $\left(k_{2}^{+}(\alpha)\right)^{\prime},\left(c_{2}^{+}(\alpha)\right)^{\prime},\left(k_{1}^{-}(\alpha)\right)^{\prime}$ and $\left(c_{1}^{-}(\alpha)\right)^{\prime}$ are negative numbers. The "prime" denotes differentiation with respect to $\alpha$. Then there is a $\lambda>0$ so that $\left(k_{1}^{+}(\alpha)\right)^{\prime}=\left(c_{1}^{+}(\alpha)\right)^{\prime}=\left(k_{2}^{-}(\alpha)\right)^{\prime}=\left(c_{2}^{-}(\alpha)\right)^{\prime}=\lambda$ and $\left(k_{2}^{+}(\alpha)\right)^{\prime}=\left(c_{2}^{+}(\alpha)\right)^{\prime}=\left(k_{1}^{-}(\alpha)\right)^{\prime}=\left(c_{1}^{-}(\alpha)\right)^{\prime}=-\lambda$. Then, for the $(2-S)$ exist we need

$$
\begin{aligned}
& \frac{\partial u_{1}^{+}}{\partial \alpha}=\frac{\partial u_{2}^{-}}{\partial \alpha}=\lambda(\sin (x)(\cosh (t)+\sinh (t))+\cos (x)(\cosh (t)-1+\sinh (t)))>0 \\
& \frac{\partial u_{2}^{+}}{\partial \alpha}=\frac{\partial u_{1}^{-}}{\partial \alpha}=-\lambda(\sin (x)(\cosh (t)+\sinh (t))+\cos (x)(\cosh (t)-1+\sinh (t)))<0
\end{aligned}
$$

Since (6.3) holds for each $t \in[0, M]$ and $x \in\left(0, \frac{\pi}{2}\right)$ therefore, $\tilde{U}^{i}(t, x)$ is $(2-S)$ and

$$
\tilde{U}^{i}(t, x)=\tilde{C}^{i} \cosh (t) \sin (x)-\tilde{C}^{i} \sinh (t) \sin (x)+\tilde{K}^{i} \cos (x)(\cosh (t)-1)-\tilde{K}^{i} \sinh (t) \cos (x)
$$

for all $t \in[0, M]$ and $x \in\left(0, \frac{\pi}{2}\right)$.

\section{RÉFÉRENCES}

[1] Atanassov, K. (1986) Intuitionistic fuzzy sets, Fuzzy Sets and Systems, 20, 87-96.

[2] G.S. Mahapatra, T.K. Roy, Intuitionistic Fuzzy Number and Its Arithmetic Operation with Application on System Failure Journal of Uncertain Systems Vol.7, No.2, pp.92-107, 2013

[3] Atanassov, L. Vassilev, P.Tsvetkov, R. (2013) Intuitionistic Fuzzy Sets, Measures and integrals Bulgarian Academic Monographs

(12), Professor Marin Drinov Academic Publishing House, Sofia.

[4] L. S. Chadli, A. Harir and S. Melliani, Fuzzy Euler differential equation, SOP Transactions on Applied Mathematics, volume 2, number 1, January 2015

[5] L. S. Chadli, A. Harir and S. Melliani, Solutions of fuzzy heat-like equations by variational iterative method , Annals of Fuzzy Mathematics and Informatics, Volume 10, number 1, 2015, pp 29-44.

[6] L. S. Chadli, A. Harir and S. Melliani, Solutions of fuzzy wave-like equations by variational iteration method. International Annals of Fuzzy Mathematics and Informatics, volume 8, number 4, 2014, 527-547 .

[7] J.H. He, Variational iteration method-A kind of nonlinear analytical technique : some examples, International Journal of NonLinear Mechanics 34 (1999) 699-708.

[8] J.H He, Variational principles for some nonlinear partial differential equations with variable coefficients. Chaos, Solitons and Fractals 19(2004) 847-851.

[9] S. Melliani and L. S. Chadli, Introduction to intuitionistic fuzzy partial differential equations, Notes on Intuitionistic Fuzzy Sets Vol. 8 (2001), No. 1, pp. 24-28.

[10] A.K. Shaw and T. K. Roy, Some arithmetic operations on Triangular Intuitionistic Fuzzy Number and its application on reliability evaluation, International Journal of Fuzzy Mathematics and Systems. ISSN 2248-9940 Volume 2, Number 4 (2012), pp. 363-382

[11] AM. Wazwaz, A.Gorguis, Exact solutions for heat-like and wave-like equations with variable coefficients, Appl. Math. Comput. $149(2004) 15-29$.

[12] L.A. Zadeh, Fuzzy sets, Inform. and Control 8 (1965) 338-353 
(A.HARIR) Laboratory of Applied Mathematics and Scientific Computing, Sultan Moulay Slimane University, P.O. Box 523, Beni Mellal, 23000

Current address: Laboratory of Applied Mathematics and Scientific Computing, Sultan Moulay Slimane University, P.O. Box 523, Beni Mellal, 23000

E-mail address, A.Harir: atimad.harir@gmail.com

(S. Melliani) Laboratory of Applied Mathematics and Scientific Computing, Sultan Moulay Slimane University, P.O. Box 523, Beni Mellal, 23000

E-mail address, S. Melliani: s.melliani@usms.ma

(L.S.Chadli) Laboratory of Applied Mathematics and Scientific Computing, Sultan Moulay Slimane University, P.O. Box 523, Beni Mellal, 23000

E-mail address, L.S.Chadli: sa.chadli@yahoo.fr 